\title{
LANDING ASSISTANCE AND EVALUATION USING IMAGE PROCESSING
}

Kalyani Deshmukh *1, S. D. Mali ${ }^{2}$

${ }^{* 1}$ Student, Electronics Department, Sinhgad College of Engineering, Pune, INDIA

${ }^{2}$ Associate Professor, Electronics and telecommunication Department, Sinhgad College of Engineering, Pune, INDIA

\section{Abstract:}

Most of the landing systems are based on GPS and radar altimeter, sonar, infrared. But in urban environments buildings and other obstacles disturb the GPS signal and can even cause loss of signal. In such case it will be beneficial to have independent control of navigation and landing assistance system. So the main aim is to design a software system that will assist helicopter or Unmanned Aerial Vehicle accurately under all-weather. The software system takes height parameter and images from helicopter or Unmanned Aerial Vehicle as an input. After applying number of processing techniques like edge detection, RGB to Gray scale on the image, the image is compared with the HSV dataset to find the free space. For edge detection Canny edge detection algorithm is used. From the number of free spaces nearest patch is selected by taking vehicle dimension and landing orientation of the vehicle into consideration. Performance of the system depends on the accuracy and the speed of the system. This system also resolves the potentially dangerous problem of GPS denial.

Keywords:

Unmanned Aerial Vehicle (UAV), Canny Egge Detection, Globle Positioning System (GPS)

Cite This Article: Kalyani Deshmukh, and S. D. Mali, "LANDING ASSISTANCE AND EVALUATION USING IMAGE PROCESSING” International Journal of Research - Granthaalayah, Vol. 3, No. 6(2015): 84-92. DOI: 10.29121/granthaalayah.v3.i6.2015.3003.

\section{INTRODUCTION}

The specific task focused in this paper is the landing of an Unmanned Aerial Vehicle or helicopter in an uncontrolled environment. In this situation, landing site cannot be carefully preselected. Due to power constraints and the desire to avoid detection we do not use radar or other active range sensing devices. Instead of this we propose a method for autonomous landing based on principles of computer vision.

Many autonomous landing systems for Unmanned Aerial Vehicles (UAVs) and helicopters are based on GPS and a dedicated close range sensor for accurate altitude measurement (radar altimeter, sonar, infrared). But, in urban environments buildings and other obstacles disturb the GPS signal. This can cause loss of signal. Therefore, only with independent control of navigation and landing guidance system can meet the requirements. For this purpose of implementing 


\section{INTERNATIONAL JOURNAL of RESEARCH -GRANTHAALAYAH

UAV's and helicopter's landing automatically and accurately under all-weather, a new program of UAV's automatic landing is proposed in this paper.

Namely we are giving height parameter and aerial image as an input. After performing various operations or using various algorithm, we will get labeled blocks. From that labeled blocks and dimensions of UAV or helicopter we will find the best place for landing which is nearer to it. After finding the best nearest patch for landing. In this paper, we select a safe landing site from elevation and appearance, avoiding trees and tall grasses and drive the UAV to the selected landing site. The overall landing system consists of a low-level vision subsystem which recovers a ground elevation map, a map building component which classifies ground objects using appearance and the recovered topography, and a high-level navigation system for specifying way-points to the desired landing target.

\section{RELATED WORK}

Guili Xu, Yong Zhang, Shengyu Ji, Yuehua Cheng, Yupeng Tian, [1].

In this paper, a novel approach to UAV's automatic landing on the ship's deck is proposed.. The infrared radiation images are used in the experiments by extracting the target from the background to analyze and then recognizing it. Also, the angle of yaw is calculated. The average recognition time is $17.2 \mathrm{~ms}$ which is obtained through the use of affine moment invariants. This type of speed is expected to improve the reliability and real-time performance of autonomous UAV landing.

Srikanth Saripalli, James F. Montgomery and Gaurav S. Sukhatme, [2].

This paper present the design and implementation of a real time, vision-based landing algorithm for an autonomous helicopter. The helicopter is required to navigate from an initial position to a final position in a partially known environment based on GPS and vision, locate a landing target (a helipad of a known shape) and land on it.

Courtney S. Sharp Orriid Shakernia S. Shankar Sastry, [3].

In this paper the design and implementation of a real-time computer vision system for a rotorcraft unmanned aerial vehicle to land onto a known landing target is presented. This vision system consists of customized software and off-the-shelf hardware which perform image processing, segmentation, feature point extraction, camera pan/tilt control, and motion estimation.

Scott M. Ettinger, Michael C. Nechyba Peter G. Ifju Martin Waszak, [4]

Recently, substantial progress has been made towards designing, building and test-flying remotely piloted Micro Air Vehicles (MAVs). This progress in overcoming the aerodynamic obstacles to flight at very small scales has, unfortunately, not been matched by similar progress 


\section{INTERNATIONAL JOURNAL of RESEARCH -GRANTHAALAYAH

in autonomous MAV flight. Thus, a robust, vision-based horizon detection algorithm as the first step towards autonomous MAVs was proposed in this paper.

Fitzgerald, Daniel L. and Walker, Rodney A. and Campbell, Duncan A, [5].

In this paper, a vision-based terrain mapping and analysis system, and a model predictive control (MPC) - based flight control system, for autonomous landing of a helicopter-based unmanned aerial vehicle (UAV) in unknown terrain is presented. The vision system is centered around Geyer et al.'s Recursive Multi-Frame Planar Parallax algorithm, which accurately estimates 3D structure using geo referenced images from a single camera, as well as a modular and efficient mapping and terrain analysis module.

Tiago F. Gonc,alves, Jos`e R. Azinheira Tiago F. Gonc alves, Jos’e R. Azinheira, [6].

This paper presents a feasibility study of a vision-based autonomous approach and landing for an aircraft using a direct visual tracking method. Auto-landing systems based on the Instrument Landing System (ILS) have already proven their importance through decades but general aviation stills without cost-effective solutions for such conditions.

\section{PROPOSED WORK}

The proposed Landing Assistance System first checks height parameter of the UAV or helicopter. Simultaneously it takes aerial images from their camera. From the height parameter of the UAV or helicopter, block size is estimated. After block size estimation, block is generated with the help of aerial images and block size. Next Step is RGB to Gray Scale conversion, in this we convert original image to the gray scale image because we need gray scale image as an input to the Canny Edge Detection.

After RGB to Gray Scale conversion, our next step is Edge Detection. Edge Detection is done on the image to find the edges in the image. By edge detection, objects in the images is find out. If the object is there means that space is not free for landing. So Edge Detection is necessary to determine the free space. After that blocks are generated to find large free space. Means if the edge is there in the block then that block will be skipped and more number of blocks which are connected together are taken into consideration for free space.

Simultaneously, Input Aerial Image is passed through RGB to HSV conversion RGB to HSV conversion is done because we want to give the range of colour in the image. HSV only allows us to give range of colours in the image. In the next step the output of block generation is compared with the HSV image using HSV dataset. So as to get labeled blocks. In this we need to separate all the colours according to its feature like shades of green colour is grass or tree and shades of blue colour is water and likewise. In this step all the feature set is generated which will help us to find the space for landing. It will separate all the component form the image like water, grass, land etc. 


\section{INTERNATIONAL JOURNAL of RESEARCH -GRANTHAALAYAH

From the labeled block and UAV dimensions, whichever places is suitable for landing will be find out find out. If any orientation variations will be there then it will again sort out the best places for landing from it. From the places it will again sort out whichever place is nearer to the UAV or helicopter for landing. And finally it will guide the UAV or helicopter to land on the nearest patch.

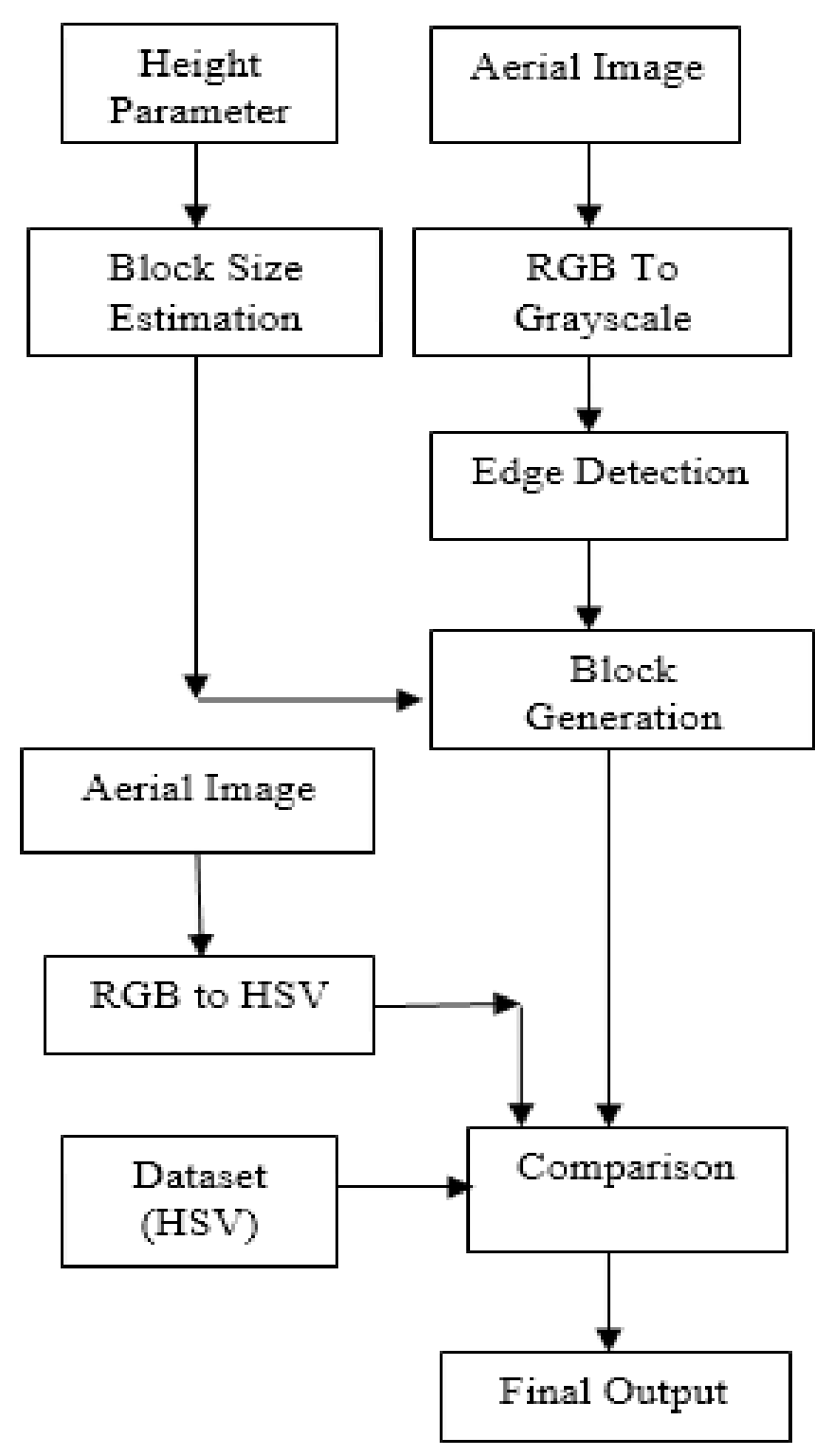

Figure 1: Block Diagram of Landing Assistance and Evaluation 


\section{INTERNATIONAL JOURNAL of RESEARCH -GRANTHAALAYAH

\section{METHODOLOGY}

\section{EDGE DETECTION}

Edge detection is a basic tool used in image processing, basically for feature detection and extraction. The aim is to identify points in a digital image where brightness of image changes sharply and find discontinuities. The purpose of edge detection is reducing the amount of data in an image and preserves the structural properties for further image processing. In a grey level image, the edge is a local feature, within a neighborhood. It separates each region, where the gray level is more or less uniform with the different values on the two sides of the edge. For a noisy image, it is difficult to detect edges as both edge and noise contains high frequency contents which results into blurred and distorted result.

Many edge detection techniques have been developed for extracting edges from digital images. Gradient based classical operators like Robert, Prewitt, Sobel were initially used for edge detection. But they are not able to detect sharp edges and also highly sensitive to noise image. Laplacian based Marr Hildrith operators also suffers from two limitations i.e. High probability of detecting false edges and the localization error may be severe at curved edges[10]. Canny edge detection algorithm was proposed by John F. Canny in 1986. It is considered as the ideal edge detection algorithm for images that are corrupted with noise. Canny's aim was to discover the optimal edge detection algorithm which reduces the probability of detecting false edge, and gives sharp edges. In the optimal edge detection algorithm, an "optimal" edge detector means:

$>$ Good detection - the algorithm should mark as many real edges in the image as possible.

$>$ Good localization - edges marked should be as close as possible to the edge in the real image.

$>$ Minimal response - a given edge in the image should only be marked once and image noise should not create false edges.

\section{Canny Edge Detection Algorithm:}

\section{STEP I: Noise reduction by smoothing}

Noise contained in image is smoothed by convolving the input image I $(i, j)$ with Gaussian filter G. Mathematically, the smooth resultant image is given by

$$
F(i, j)=G * I(i, j)
$$

Prewitt operators are simpler to operator as compared to sobel operator but more sensitive to noise in comparison with sobel operator [10].

\section{STEP II: Finding gradients}

In this step we detect the edges where the change in grayscale intensity is maximum.Required areas are determined with the help of gradient of images. Sobel operator is used to determine the gradient at each pixel of smoothened image. Sobel operators in $\mathrm{i}$ and $\mathrm{j}$ directions are given as 


\section{INTERNATIONAL JOURNAL of RESEARCH -GRANTHAALAYAH

$$
\begin{aligned}
& D i=\left[\begin{array}{rrr}
-1 & 0 & +1 \\
-2 & 0 & +2 \\
-1 & 0 & +1
\end{array}\right] \\
& \operatorname{Dj}=\left[\begin{array}{rrr}
+1 & +2 & +1 \\
0 & 0 & 0 \\
-1 & -2 & -1
\end{array}\right]
\end{aligned}
$$

These sobel masks are convolved with smoothed image and giving gradients in $\mathrm{i}$ and $\mathrm{j}$ directions.

$$
\mathrm{Gi}=\mathrm{Di} * \mathrm{~F}(\mathrm{i}, \mathrm{j})
$$

$\mathrm{Gj}=\mathrm{Dj} * \mathrm{~F}(\mathrm{i}, \mathrm{j})$

Therefore edge strength or magnitude of gradient of a pixel is given by

$$
\mathrm{G}=\sqrt{G i^{2}+G j^{2}}
$$

The direction of gradient is given by

$$
\theta=\arctan \left(\frac{G j}{G i}\right)
$$

$\mathrm{Gi}$ and $\mathrm{Gj}$ are the gradients in the $\mathrm{i}$ and $\mathrm{j}$ directions respectively.

\section{STEP III: Non maximum suppressions:}

Non maximum suppression is carried out to preserves all local maxima in the gradient image, and deleting everything else this results in thin edges.

For a pixel M (i,j):

- Firstly round the gradient direction nearest to $45^{\circ}$, then compare the gradient magnitude of the pixels in positive and negative gradient directions i.e. If gradient direction is east then compare with gradient of the pixels in east and west directions. let $\mathrm{E}(\mathrm{i}, \mathrm{j})$ and $\mathrm{W}(\mathrm{i}, \mathrm{j})$ respectively.

- If the edge strength of pixel $M(i, j)$ is largest than that of $E(i, j)$ and $W(i, j)$, then preserve the value of gradient and mark $\mathrm{M}(\mathrm{i}, \mathrm{j})$ as edge pixel, if not then suppress or remove.

\section{STEP IV: Hysteresis thresholding:}

The output of non-maxima suppression still contains the local maxima created by noise. Instead choosing a single threshold, for avoiding the problem of streaking two thresholds t_high and t_low are used.

For a pixel $\mathrm{M}(\mathrm{i}, \mathrm{j})$ having gradient magnitude $\mathrm{G}$ following conditions exists to detect pixel as edge:

$>$ If $\mathrm{G}<\mathrm{t}$ _low than discard the edge.

$>$ If $\mathrm{G}>$ than $\mathrm{t} \_$high keep the edge. 


\section{INTERNATIONAL JOURNAL of RESEARCH -GRANTHAALAYAH

$>$ If $\mathrm{t}$ _low $<\mathrm{G}<\mathrm{t}$.high and any of its neighbors in a $3 \times 3$ region around it have gradient magnitudes greater than $t \_$high, keep the edge.

$>$ If none of pixel $(\mathrm{x}, \mathrm{y})$ 's neighbors have high gradient magnitudes but at least one fall between t_low and t_high search the $5 \times 5$ region to see if any of these pixels have magnitude greater than thigh. If so, keep the edge.

Else, discard the edge.

\section{Advantages of canny edge detection algorithm:}

1. Less Sensitive to noise.

2. Remove streaking problem.

3. Adaptive in nature.

4. Good localization.

\section{RESULTS AND DISCUSSION}

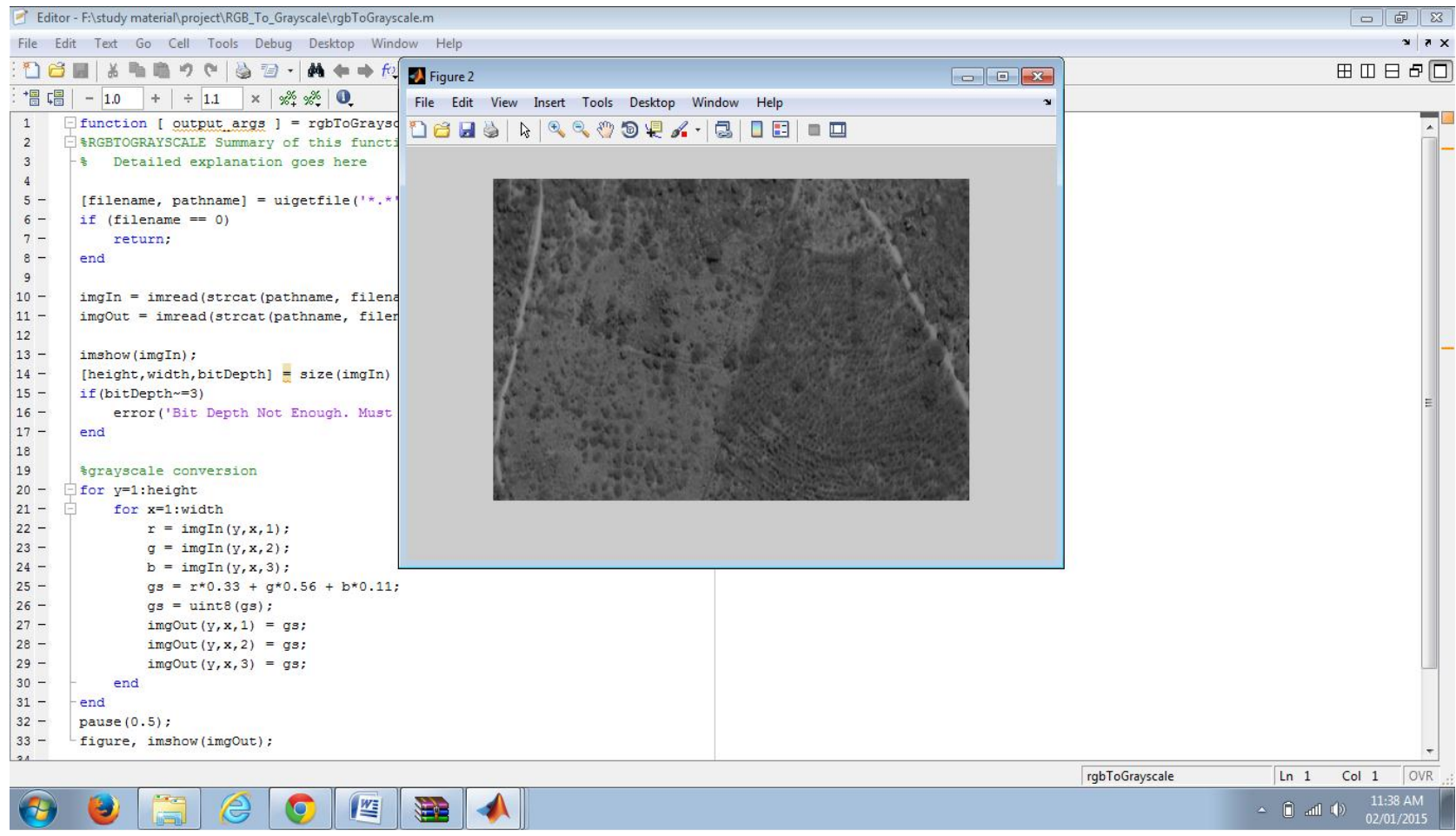

Figure 2: Output of Gray scale image is given as an input to Canny Edge Detection and Blacks are generated in the output image of Canny Edge Detection to find the free space. 


\section{INTERNATIONAL JOURNAL of RESEARCH -GRANTHAALAYAH \\ A knowledge Repository}

Science

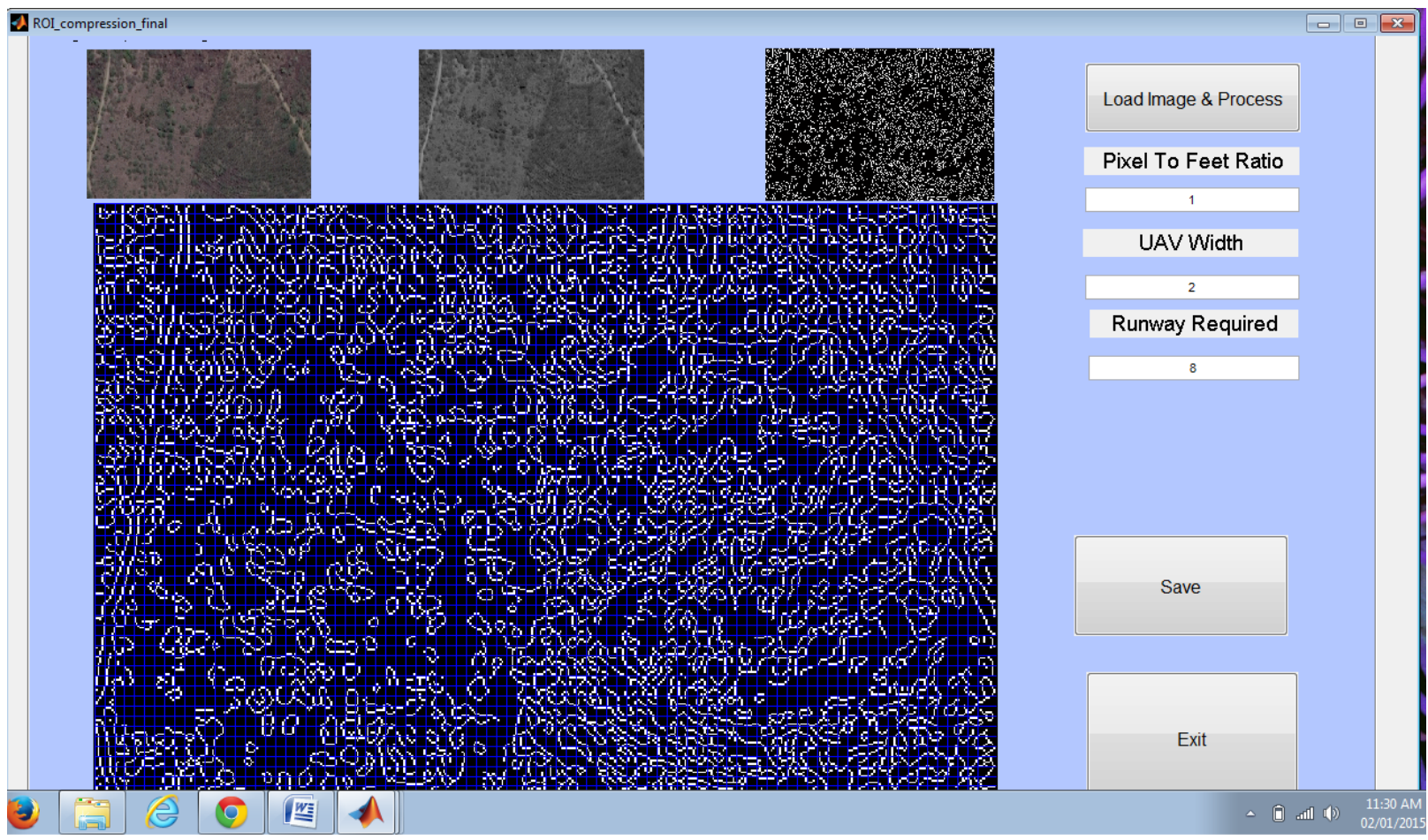

Figure 3: Result of Canny Edge Detection and Block Generation

\section{CONCLUSIONS}

Better results are obtained using Canny Edge detection as other operators like Sobel, Robert, Prewitt are unable to detect sharp edges and also highly sensitive to the noise images. Canny edge detection algorithm reduces the probability of detecting false edge, and gives sharp edges.Also in the project, we will study the basic research concerning automatic UAV navigation and landing. By extracting the target from the background, we will analyze the infrared radiation images in our experiments.

\section{ACKNOWLEDGEMENTS}

Any accomplishment requires the effort of many people and this work is no different. I find great pleasure in expressing my deep sense of gratitude towards all those who have made it possible for me to present this work. I would like to the express my true sense and a sincerest gratitude to my guide Prof. S. D. Mali for his dynamic and valuable guidance. I am grateful to him for constant encouragement in the fulfillment of the project work. This project is a result of combined efforts put in by my guide and me. I would also like to thank him for providing me with all necessary infrastructure and facilities to complete the project work. 


\section{INTERNATIONAL JOURNAL of RESEARCH -GRANTHAALAYAH

\section{REFERENCES}

[1] Guili Xu, Yong Zhang, Shengyu Ji, Yuehua Cheng, Yupeng Tian, "Research on computer vision-based for UAV autonomous landing on a ship",Science Direct journal homepage: www.elsevier.com/locate/patrec,2009.

[2] Srikanth Saripalli, James F. Montgomery and Gaurav S. Sukhatme, "Vision-based Autonomous Landing of an Unmanned Aerial Vehicle”, IEEE International Conference on Robotics and Automation pp 2799-2804, May 2002., Washington D.C., USA2002.

[3] Courtney S. Sharp Orriid Shakernia S. Shankar Sastry, "A Vision System for Landing an Unmanned Aerial Vehicle”, IEEE International Conference on Robotics \& Automation Seoul, Korea. May 21-26, 2001.

[4] Scott M. Ettinger, Michael C. Nechyba Peter G. Ifju Martin Waszak, "Towards Flight Autonomy: Vision-Based Horizon Detection for Micro Air Vehicles”.

[5] Fitzgerald, Daniel L. and Walker, Rodney A. and Campbell, Duncan A, "A Vision Based Emergency Forced Landing System for an Autonomous UAV”, Australian International Aerospace Congress Conference, Melbourne Australia, 2005.

[6] Tiago F. Gonc,alves, Jos'e R. Azinheira Tiago F. Gonc,alves, Jos'e R. Azinheira, "Vision-Based Autonomous Approach And Landing For An Aircraft Using A Direct Visual Tracking Method”, IDMEC, IST/TULisbon, Av. Rovisco Pais N.1, 2004.

[7] Flusser, J., Kautsky, J., Sroubek, F., "Object recognition by implicit invariants Computer analysis of images and patterns”, In: Proc. 12th Internat. Conf. CAIP 2007, Vienna, Austria, pp. 856-863.

[8] Ettinger, Scott M., Nechyba, C., lfju, Peter G., et al., "Towards Flights autonomy: Vision-based horizon detection for micro air vehicles”, IEEE Internat. Conf. on Robotics and Automation, Washington DC.

[9] Sharp, Courtney S., Shakemia, Omid, Sastry, Shankar S., "A vision system for landing an unmanned aerial vehicle”, In: Proc. 2001 ICRA. IEEE Internat. Conf. on Robotics and Automation, Seoul, South Korea, pp. 1720-1727.

[10] Saripalli, Srikanth, Montgomery, James F., Sukhatme, Gaurav S.,. "Vision-based autonomous landing of an unmanned aerial vehicle”, In: Proc. 2002 IEEE Internat. Conf. on Robotics and Automation, Washington, DC, USA, pp. 2799- 2804.

[11] Heerman P.D. and N.Khazenie, "Classification of multispedtral remote sensing data using a bach propagation neural network”, IEEE Trans, Geosci. Remote Sensing, vol.GE_30,no.1,pp.81-88.

[12] Cesetti A., Frontoni, E., Mancini, A., Zingaretti P, "Autonomous safe landing of a vision guided helicopter", IEEE International Conference on Mechatronics and Embedded Systems and Applications (MESA), 2012. 